Mitteilungen der Österreichischen Geographischen Gesellschaft, 160. Jg., S. 360-370

(Annals of the Austrian Geographical Society, Vol. 160, pp. 360-370)

Wien (Vienna) 2018, https://doi.org/10.1553/moegg160s360

\title{
WILHELM VON TEGETTHOFF 1827-1871 - GLOBALE INTERESSEN UND MOBILITÄT SEIT DER MitTE DES 19. JAHRHUNDERTS
}

\author{
Robert KostKa, Graz* \\ mit 4 Abb. im Text
}

\section{INHALT}

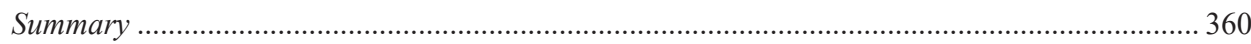

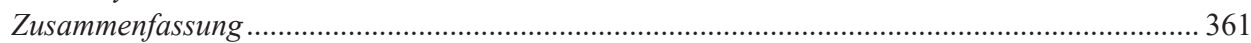

1 Wilhelm von Tegetthoff und seine steirische Heimat ...................................................... 361

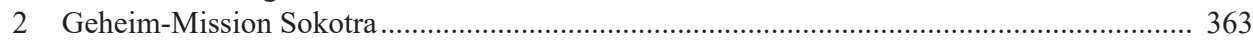

3 Über die Globalität von Reisen, die mit dem Namen Tegetthoff in Beziehung

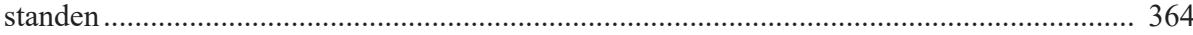

4 Franz-Josef-Land und der Name Tegetthoff bis zur Gegenwart......................................... 366

5 Globalität und Mobilität der Gegenwart am lokalen Beispiel des Tegetthoff-Steines ........... 367

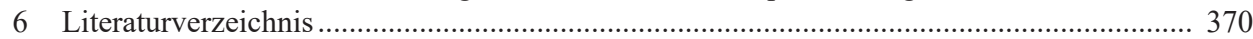

\section{Summary}

\section{Wilhelm von TegetThoff 1827-1871 - Global InTERESTS AND Mobility SINCE THE MidDle OF THE $19^{\mathrm{TH}}$ CENTURY}

The increasing globality and mobility since the second half of the $19^{\text {th }}$ century influenced the life and the environment of Wilhelm von Tegetthoff. In addition his name did not fall into oblivion until now. Wilhelm von Tegetthoff was born in 1827 in Maribor, at that time Marburg an der Drau in Styria. He died in 1871 and was buried at St. Leonhard cementary in Graz. In 1870 a memorial, the "Tegetthoff-stone", was erected for him at the southern slope of the Schöckl mountain near Graz.

With the help of Archduke Ferdinand Maximilian he could follow his interests in seafaring and marine and became admiral in this field. Numerous global places and journeys were connected with his interests, among which there were a visit of the island of Socotra in Yemen, long distance travels with the fregatte "Novara", Mexico 1864-1867, the opening of the Suez channel 1869 and others.

In the frame of the Austrian-Hungarian "Nordpolexpedition" (expedition to the northern polar region) from 1872 to 1874 the archipelago "Franz Josephs Land" was discovered. The expedition ship was given the name "Admiral Tegetthoff" and a position in this island group got the name Cape Tegetthoff, existing until today.

\footnotetext{
* Tit. Ao. Univ.-Prof. Dipl.-Ing. Dr. techn. Robert KostKa, Technische Universität Graz, Institut für Geodäsie, Steyrergasse 30, A-8010 Graz; E-Mail: ruth.reisch@tugraz.at
} 
In 1870 the "Tegetthoff-stone" at the southern slope of Schöckl was erected and is now situated in an area which is used today by modern townspeople for tourism and sports. Changes in this area were the consequence, the historical object of the Tegetthoff-stone should be influenced by these measures as little as possible.

\section{Zusammenfassung}

Die zunehmende Globalität und Mobilität seit der zweiten Hälfte des 19. Jahrhunderts beeinflussten das Leben und die Umwelt des Wilhelm von Tegetthoff. Sein Name ist bis heute nicht in Vergessenheit geraten. Wilhelm von Tegetthoff wurde 1827 in Maribor, damals Marburg an der Drau in der Steiermark, geboren. Er verstarb 1871 und wurde am Sankt-Leonhard-Friedhof in Graz begraben. 1870 wurde für ihn eine Gedenkstätte, der ,,Tegetthoff-Stein “ am Südhang des Schöckl errichtet.

Mit Unterstützung von Erzherzog Ferdinand Maximilian konnte er sein Interesse an der Seefahrt verfolgen und brachte es bei der Marine zum Admiral. Zahlreiche globale Plätze und Reisen sowie große Mobilität waren damit verbunden wie beispielsweise ein Besuch der Insel Sokotra im Jemen, Fernreisen mit der Fregatte „Novara“, Mexiko 1864-1867, die Eröffnung des Suezkanals 1869 und andere.

Im Rahmen der Österreichisch-Ungarischen Nordpolexpedition 1872 bis 1874 wurde der Archipel Franz-Josef-Land entdeckt. Das Expeditionsschiff erhielt den Namen „Admiral Tegetthoff" und einer Position auf der Inselgruppe wurde der Name „Kap Tegetthoff“, der heute noch existiert, gegeben.

Der 1870 errichtete „,Tegetthoff-Stein “ am Südabhang des Schöckl liegt heute in einem Gebiet, das zurzeit von den modernen Stadtmenschen für touristische und sportliche Aktivitäten genützt wird. Veränderungen sind die Folge, das historische Objekt des Tegetthoff-Steines sollte durch Maßnahmen aber möglichst wenig beeinflusst werden.

\section{Wilhelm von Tegetthoff und seine steirische Heimat}

Am Südabfall des Grazer Hausberges Schöckl (1445 m) befindet sich, etwas unter der Seilbahn oberhalb der Ortschaft St. Radegund, ein Aussichtspunkt mit einem umfassenden Rundblick in den Süden/Südosten der Steiermark. Das Besondere an dieser Stelle ist ein Gedenkstein für Wilhelm von Tegetthoff, der diese Stelle in seinen späten Jahren häufig aufgesucht hat. Dieser Stein, eine kleine Säule von etwa zwei Metern Höhe, enthält eine gravierte Inschrift aus dem Jahr 1870, die auch heute noch deutlich zu erkennen ist: „Hier hat 1870 Tegetthoff oft und gerne geweilt.“ Eine Führung der „Grazguides“ durch den 2. Grazer Stadtbezirk beginnt meist am dortigen Tegetthoff-Platz beim Tegetthoff-Denkmal, das hier erst nach einer längeren Reise seine Bleibe gefunden hat.

Wilhelm von Tegetthoff wurde 1827 in Marburg an der Drau, damals noch im Kronland Steiermark der Österreichisch-Ungarischen Monarchie, geboren. Sein aus Westfalen stammender Vater wurde in den erblichen Adelsstand erhoben, seine Mutter war aus Graz. Der Lebensweg Wilhelm von Tegetthoffs wurde entscheidend durch sein Verhältnis zu Erzherzog Ferdinand Maximilian (1832-1867), dem jüngeren Bruder von Kaiser Franz Joseph, geprägt, der damals als Vize-Admiral das Oberkommando über die S. M. Kaiserliche Marine innehatte.

Nach Abschluss der Schulzeit besuchte Tegetthoff das Marine Kollegium und wurde bereits früh bei vielen Fahrten und Expeditionen der kaiserlichen Marine eingesetzt. Durch überdurchschnittliche Leistungen und diplomatische, militärische und organisatorische Fähigkeiten machte er auf sich aufmerksam. So erhielt er bereits 1859 das Kommando über ein Schiff, das Erzherzog Ferdinand Maximilian nach Brasilien führte. 
Im Jahr 1868 erfolgte seine Ernennung zum Marinekommandanten, danach zum Marineoberkommandanten und zum Admiral. Seine Aufgaben (Reformvorhaben) bestanden nunmehr in organisatorischen Maßnahmen, die bis zum Ende der Doppelmonarchie 1918 in Kraft blieben.

Im Frühjahr 1871 erkrankte Tegetthoff in Wien an einer Lungenentzündung. Sein Gesundheitszustand war schon zuvor nicht zufriedenstellend gewesen und wurde von einem Internisten in seiner Heimatstadt Graz behandelt. Dieser riet ihm zu einem Aufenthalt im Kurort St. Radegund am Fuß des Schöckl. Dort bezog er ein Quartier im hochgelegenen Ortsteil. Noch heute ist die „Tegetthoff Villa“, von der er immer wieder Ausflüge unternehmen konnte, ein Begriff.

Am 7. April 1871 verstarb Wilhelm von Tegetthoff in Wien unter starker Anteilnahme der damaligen Medien. Er wurde mit hohen militärischen Ehren in Wien begraben. Kaiser Franz Joseph konnte an der Beerdigung wegen eines Aufenthalts in Meran aber nicht teilnehmen. Am 31. Oktober 1872 erfolgte schließlich die Überführung des Sarkophags in seine Heimatstadt Graz auf den St.Leonhard-Friedhof, der seither die letzte Ruhestätte Wilhelm von Tegetthoffs ist.

Am Familiengrab (Abb. 1) ist unter dem Wappen von Maria Theresia mit den Initialen M T eingraviert: Carl v. Tegetthoff; Wilhelm v. Tegetthoff, k.k. Vizeadmiral geb. 23. Decb. 1827 gest. 7. April 1871; Albrecht v. Tegetthoff; Carl v. Tegetthoff; Leopoldine v. Tegetthoff, Witwe.

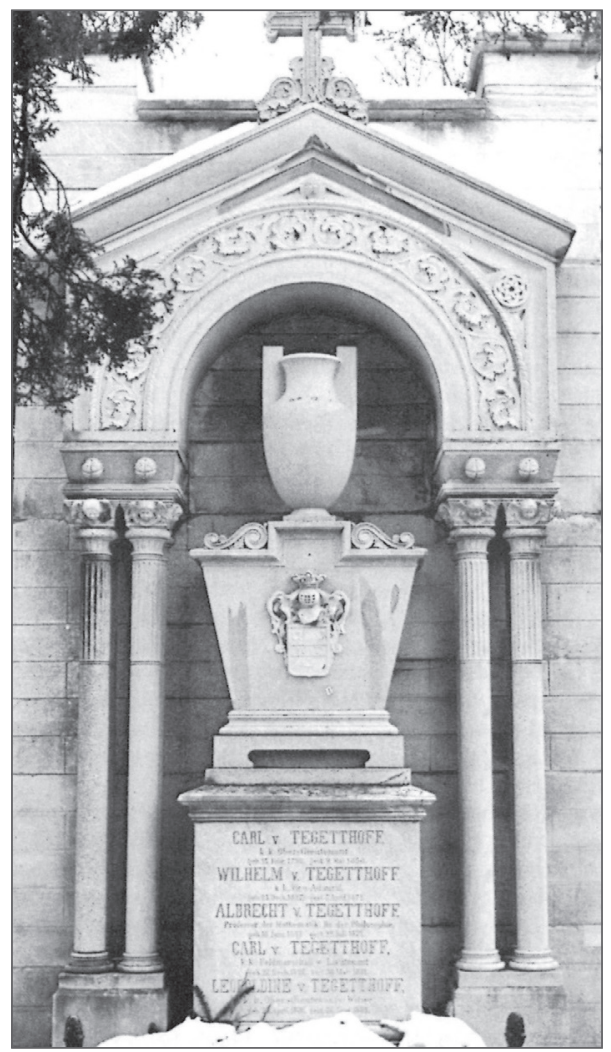

Quelle: BASCh-Ritter 1987

Abb. 1: Grabstätte der Familie Tegetthoff im Friedhof St. Leonhard in Graz 


\section{Geheim-Mission Sokotra}

Eines der politischen Ziele von Erzherzog Ferdinand Maximilian war der Ausbau der Kriegsund Handelsflotte, die die Macht des Habsburgerreiches auch nach Übersee tragen könnte. Dazu hatte er vorgesehen, dass Wilhelm vom Tegetthoff in geheimer Mission nach Südarabien und zum Horn von Afrika reisen und die Insel Sokotra (Socotra) auf ihre Eignung als Landungsplatz der österreichischen Marine erforschen sollte. Er vermerkte dazu in geraffter Form:

„Vom 2.4.1857 bis zum 24.4.1858 auf Urlaub in Ägypten. Mit nächstem Lloyd Dampfer haben Sie sich nach Alexandrien zu begeben, um eine Mission anzutreten. [...] Der Suezkanal wird Realität. Will Österreich Weltmacht sein, so müssen Stützpunkte an der Küste des Roten Meeres oder im Süden von Arabien erworben werden. Diese Mission sollte so geheim wie möglich gehalten und als Privatsache angegeben werden. Geheim gehalten werden sollte vor allem der Zweck der Mission. Der durch den geplanten Suezkanal gelangende Handel mit Indien und dem übrigen Asien und schließlich selbst noch die Notwendigkeit für Österreich, ein Besitztum für Deportation zu besitzen, machten es wünschenswert, im Roten Meer eine Insel oder einen Küstenstrich zu besitzen. Kauf, Schenkung oder eine andere Weise, wie Österreich zu einem Eigentumsrecht gelangen könnte, sollte erkundet werden. Von vorrangigem Interesse sei die Insel Socotra zu erkunden, auf welche Weise man am besten, leichtesten und sichersten in Besitz derselben gelangen könnte. " (JANATA 1989, S. 23)

In einem Tagebuch gab Tegetthoff den Verlauf der Reise, das Ergebnis der Mission und Empfehlungen an (JANATA 1989). Nach längerer Wartezeit auf seinen Reisebegleiter Dr. Heuglin in Kairo ging die Reise in Afrika weiter. 20 Tage den Nil aufwärts waren erfreulich, der sechstägige Kamelritt vom Niltal durch die Wüste bis zum Roten Meer weniger. Die Qual des Kamelritts zum Roten Meer und zu einem Küstensegler, zu Ankerplätzen und Häfen sowie ein Missgeschick mit Kampfszene, Gefangennahme und Lösegeld, bis man endlich Aden erreichte, wurden erwähnt, ebenso Weihnachten in dieser Stadt und die Aussicht auf Weiterreise. Am 2. Februar 1858 erreichte Tegetthoff Hadebo, den Hauptort der Insel Sokotra. Es folgten Reisen im Inneren der Insel auf Kamelrücken oder als Wanderung, weiters Direktverhandlungen mit dem Sultan ohne fixe Zusagen, der Ankaufspreis schien aber akzeptabel. Es gab zwar finanzielle Probleme, aber auch Aussicht auf den Erwerb der Insel, den man später, wenn nötig, auch mit Gewalt durchführen könnte.

Eine Übersichtskarte des Jemen aus dem Jahr 1989 zeigt die Lage der Insel Sokotra östlich des Horns von Afrika und am Eingang zum Golf von Aden (Abb. 2).

Am 14. Februar 1858 vermerkte Tegetthoff die Abfahrt aus Sokotra. Via Suez, Kairo und Alexandria (Alexandien) landete er nach 176 Reise- und 219 Aufenthaltstagen in seinem Heimathafen Triest. Er berichtete von der lokalen Situation am Ausgang des Roten Meeres, von der Insel Sokotra, die der doppelten Anforderung einer Handelsstation und einer Verbrecherkolonie gerecht werden sollte. Die Bergbewohner besäßen zahlreiche Herden und weitere Produkte von Interesse. Er sprach dieser Situation mannigfache Vorteile zu. Erzherzog Ferdinand Maximilian übermittelte das Ergebnis dieser Mission an Kaiser Franz Joseph. Tegetthoff regte den Kauf der Insel an, doch Wien zeigte sich nicht interessiert. So wurde das Eiland 1878 britisch. Wegen der Geheimhaltung der Mission konnte keine öffentliche Auszeichnung Tegetthoffs erfolgen, sondern lediglich eine allerhöchste Remuneration. Der Vorschlag für diese wurde am 28. Juni 1858 von Kaiser Franz Joseph genehmigt, die verfasste Laudatio trägt den eigenhändigen Vermerk des Kaisers ,ad acta“. 


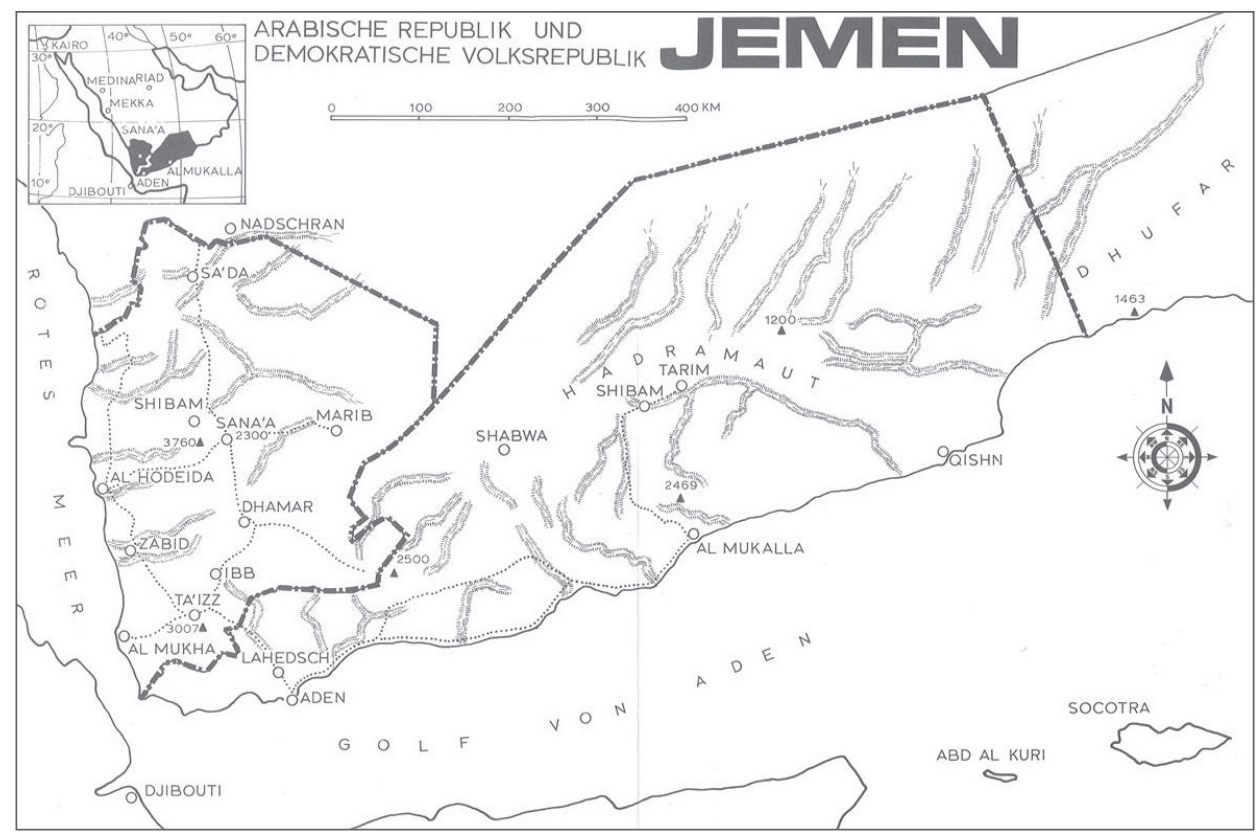

Quelle: JANATA 1989; Entwurf KostKa 1989

Abb. 2: Übersichtskarte des Jemen (Schwarz-Weiß-Verkleinerung des Originals) mit der Lage der Insel Sokotra

\section{3 Über die Globalität von Reisen, die mit dem Namen Tegetthoff in Beziehung standen}

Auf die militärischen Erfolge Tegetthoffs, wie etwa die Kampfhandlungen bei Helgoland im Jahr 1864 oder die erfolgreiche Seeschlacht bei Lissa 1866, zu denen zahlreiche Veröffentlichungen vorliegen, wird in diesem Beitrag nicht näher eingegangen. Nach dem Sieg in der Seeschlacht bei Lissa [kroat. Vis] gegen Italien erfolgte eine Inspektionsreise Wilhelm von Tegetthoffs nach England und in die USA. Dabei kam es auch zu einem Aufenthalt in New Orleans. Bei seiner Rückfahrt erhielt Tegetthoff die Nachricht vom tragischen Ende von Erzherzog Ferdinand Maximilian, von 1864 bis 1867 als Maximilian I. Kaiser von Mexiko, dessen Leiche dort bis zum Rücktransport mit der Fregatte „Novara“ bereitgehalten wurde.

Weltpolitisch waren diese Jahrzehnte vom Imperialismus und Kolonisation geprägt. Europäische Märkte traten in Wettstreit der Machtausübung. Die Motive waren der Wunsch nach wirtschaftlicher Expansion, die Suche nach Rohstoffquellen und nach neuen Absatzmärkten. Die Gewinnung neuen Lebensraumes für die rasch anwachsende Bevölkerung zählte ebenfalls dazu.

Die Fregatte „Novara“ war durch ihre Weltumseglung bekannt geworden. Die Initiative dazu war von Erzherzog Ferdinand Maximilian ausgegangen, der bei der k.k. Kriegsmarine von 1854 bis 1861 der Vorgänger von Wilhelm von Tegetthoff als Oberkommandierender war. Als Kommandeur der aufstrebenden Kriegsmarine wollte der Erzherzog durch eine wissenschaftlichen Zwecken dienende Weltumseglungsexpedition die Weltöffentlichkeit auf die Habsburger aufmerksam machen. Neben der Förderung handelspolitischer Interessen lag der Schwerpunkt der Reise vor allem in 
wissenschaftlichen Zielsetzungen (WALLISCH 2006). Die Weltumseglung startete am 3. August 1857 in Triest, die Heimkehr dorthin erfolgte am 26. August 1859. Das Schiff wurde mit militärischen Ehren empfangen. Die wissenschaftlichen Ergebnisse waren vielfältig und wurden durch eine wissenschaftliche Kommission bearbeitet, die auch die Verantwortung für die Herausgabe des Großteils des 21 Bände umfassenden Reisewerks übernahm.

Die „Novara“ stand nach ihrer Rückkehr nun wieder für weitere Einsätze im Rahmen globaler Aufträge zur Verfügung, die nicht immer erfreulichen Ergebnissen gewidmet waren. Eine der traurigsten Schiffsreisen Tegetthoffs war wohl der Heimtransport des am 19. Juni 1867 in Mexiko als Kaiser Maximilian I erschossenen früheren Erzherzogs Ferdinand Maximilian. Der Sarkophag mit den sterblichen Überresten wurde im Auftrag der kaiserlichen Familie von Wilhelm von Tegetthoff ein halbes Jahr nach des Kaisers Tod von den neuen Machthabern in Mexiko erworben und in die Heimat Österreich zurückgebracht.

Die Historikerin BASCH-RITTER schildert die Ereignisse in Mexiko: Da Mexiko seinen umfangreichen Rückzahlungsverpflichtungen an die europäischen Gläubigerstaaten nicht nachkommen konnte, besetzten französische Truppen das Land. Eine von den Franzosen einberufene Kommission erklärte Mexiko als Kaiserreich. Die Krone sollte auf Betreiben des französischen Kaisers Napoleon III. nach dem Ergebnis einer - manipulierten - Volksabstimmung dem Bruder von Kaiser Franz Joseph, Erzherzog Ferdinand Maximilian, angeboten werden (BASCH-RitTER 1987, S. 59).

Im April 1864 empfing der Erzherzog im Schloss Miramare bei Triest die mexikanischen Vertreter, die ihm die Wahl zum Kaiser von Mexiko mitteilten. Dieser nahm die Wahl an und verzichtete auf alle Rechte im Zusammenhang mit der Erbfolge innerhalb der habsburgischen Monarchie. 7000 Österreicher begleiteten den jungen Kaiser in seine neue Heimat Mexiko. Der zukünftige Kaiser Maximilian I. kam an Bord der in voller Flaggengala erschienenen „Novara“. Ende Mai ging man in Vera Cruz, der damaligen Hauptstadt Mexikos, vor Anker. Anfang Juni hielt der Kaiser seinen Einzug in die Hauptstadt. Es folgte eine Zeit wirtschaftlicher und persönlicher Schwierigkeiten. Es kam zum Bürgerkrieg. Ein Kriegsgericht verurteilte den Habsburger zum Tode. Am 19. Juni 1867 wurde der Kaiser erschossen.

Die „Novara“, die der Erzherzog als junger Seeoffizier befehligt und mit der man ihn 1864 euphorisch nach Mexiko befördert hatte, brachte nun unter der Leitung von Wilhelm von Tegetthoff auf Halbmast die sterblichen Überreste des Kaisers wieder in seine alte Heimat, nach Triest zurück.

Ein besonderes Interesse lag für Wilhelm von Tegetthoff auch in einer noch von Erzherzog Ferdinand Maximilian angeregten Idee von „Missionsreisen“ in alle Welt und auf allen Meeren durch ein Schiff der k. u. k. Marine (BASCH-RitTer 1987, S. 77). Zumindest jedes Jahr sollten die seemännische Ausbildung vertieft und wirtschaftliche Kontakte geknüpft werden. Gesellschaftliche Veranstaltungen und Einladungen gehörten auch zur Zielsetzung. Die erste dieser Reisen fand bereits im Herbst 1868 in Richtung Ostasien statt. Ein Stab von diplomatischen sowie kommerziell und wissenschaftlich gebildeten Fachleuten stand hierfür zur Verfügung. Auch nach dem Ableben Tegetthoffs verblieb übrigens bis zur Jahrhundertwende nahezu ohne Unterbrechung ein „Missionsschiff“ in Ostasien. Sieben große Reisen wurden noch bis zum erwähnten Zeitpunkt durchgeführt.

Bereits im Jahr 1847 wurde auf Vorschlag von Fürst Metternich eine internationale Kommission zur Erarbeitung eines endgültigen Bauplans für den Suezkanal gegründet. Die Grundlage stellten französische Angaben dar. Von österreichischer Seite beauftragte man den Wasserbauingenieur Alois Negrelli, dessen Pläne schließlich von Ferdinand de Lesseps mit nur wenigen Änderungen ausgeführt wurden (WAWRIK et al. 1986). Vorarbeiten im Süden Arabiens waren bereits 1857/1858 von Wilhelm von Tegetthoff durchgeführt worden, bevor es zur internationalen Veranstaltung der Eröffnung des Verbindungskanals kam. 
An diesem Festakt nahm auch Kaiser Franz Joseph teil. Er trat diese Reise mit großem Gefolge an, zu dem auch Vizeadmiral Tegetthoff gehörte. Zuerst wählte man für die Anreise den Landweg, Eisenbahnfahrten wurden durch Kutschenfahrten abgelöst und erst das letzte Stück am Schwarzen Meer bis Konstantinopel wurde auf einer türkischen Jacht zurückgelegt. Nach einem Aufenthalt in dieser Stadt erfolgte die Weiterreise auf kaiserlichen Schiffen. Es schlossen sich einige Tage im Heiligen Land an. In Jerusalem besuchte man einige heilige Stätten, auch mit Andacht in der Grabeskirche, und das österreichische Hospiz, das von Erzherzog Ferdinand Maximilian angeregt worden war und heute noch existiert.

Der Zeitplan der Weiterreise geriet durch die schlechte Wetterlage ins Wanken. Der Kaiser, Tegetthoff und einige wenige Begleiter entschieden sich trotz der gefährlichen Lage zur Weiterfahrt. Am 15. November 1869 klarte der Himmel auf und die österreichischen Schiffe gelangten bei ruhiger See und strahlendem Wetter nach Port Said am nördlichen Ausgang des Suezkanals. Die mehrtägige Festveranstaltung zur Eröffnung der Wasserstraße, die Afrika von Asien trennt, wurde ausführlich beschrieben (BASCH-RITTER 1987). Die „Kaiserreise“ war damals in der Monarchie ein interessantes, vielbeachtetes und in den Medien aufgegriffenes Ereignis. Es war die letzte Auslandsreise für Wilhelm von Tegetthoff.

\section{Franz-Josef-Land und der Name Tegetthoff bis zur Gegenwart}

Die Nordpolarforschung war ein bedeutender Forschungszweig in der Geschichte geographischer Forschungsreisen (WALLISCH 2006). Die Österreichisch-Ungarische Nordpolexpedition 1872 bis 1874 verfolgte das Ziel, das Eismeer nach Nordosten zu erkunden und in unbekannte Regionen vorzudringen. Das Ergebnis dieser Expedition war schließlich die Entdeckung eines arktischen Archipels, dem man den Namen „Franz Josephs Land“ (Franz-Josef-Land) [heute russ. Zemlja Franca-Iosifa] gab.

Der Geograph August Petermann hatte das Interesse geweckt, eine Nordost-Passage nördlich von Sibirien bis in den pazifischen Raum voranzutreiben. 1871 wurde eine Kundfahrt in die Arktis organisiert, auf der Karl Weyprecht und Julius Payer, die späteren Expeditionsleiter der Österreichisch-Ungarischen Nordpolexpedition, bis in die nördliche Barentssee vordrangen. 1872 kam es dann noch zu einer Arktisreise des Grafen Hans Wilczek von Spitzbergen bis nach Novaja Semlja (KostKa 2016). Über die Österreichisch-Ungarische Nordpolexpedition ist vielfach berichtet worden (vgl. u. a. PaYer und Weyprecht 1874, Heeresgeschichtliches Museum Wien 1996). Die Zielsetzung der Expedition lag in der Erforschung der unbekannten Meeresteile und Länder nordöstlich von Novaja Semlja bis zur Durchfahrt in die Beringstraße und den Pazifik, die Nordost-Passage.

Die Abfahrt der Expeditionsmannschaft unter Julius Payer und Karl Weyprecht erfolgte mit dem nach Tegetthoff benannten Expeditionsschiff „Admiral Tegetthoff“, einem Segelschiff mit Hilfsmotor und einer 24-köpfigen Besatzung, am 13. Juni 1872 in Bremerhaven. Wilhelm von Tegetthoff war bereits 1871 verstorben, hatte somit keinen Kontakt mehr zur Mannschaft und zum erwähnten Archipel. Über Norwegen erreichte man Novaja Semlja, wo man am 12. August 1872 die Expedition von Graf Hans Wilczek mit seinem Expeditionsschiff „Isbjorn“ traf. Gemeinsam fuhren beide Schiffe die Westküste Novaja Semljas entlang nach Norden und trennten sich am 20. August 1872 wieder (zur Arktisforschung von Graf Wilczek siehe u. a. KostKa 2016).

Die „Admiral Tegetthoff“ setzte ihre Fahrt am 21. August 1872 nach Nordosten fort. Vom Eis gefangen, triftete man von Novaja Semlja weg. Mehr als 14 Monate war die „Admiral Tegetthoff“ vom Eis eingeschlossen und weitgehend in der Dunkelheit verborgen. Das Schiff triftete in einen trostlosen Eiswinter hinein - eine Mobilität der Untätigkeit. Nach vier Monaten, am 16. Februar 1873, ging für die Mannschaft die erste Polarnacht zu Ende. Schließlich erreichte man mehr als ein 
Jahr später, am 30. August 1873, ein unbekanntes Land, das Weyprecht „Franz-Josephs-Land“ nannte. Die gefahrvolle Odyssee der „Admiral Tegetthoff“ wurde Gegenstand zahlreicher Publikationen (vgl. u. a. Heeresgeschichtliches Museum 1996).

Als man im August 1873 endlich Land vor sich sah, war der erblickte Bergrücken noch etwa 60 bis 70 Kilometer entfernt; es war ein hoher Felssporn auf der Hall Insel. Unter Julius Payer erfolgte seine erste Besteigung und man gab ihm den Namen „Kap Tegetthoff“, den es bis heute noch hat.

Da das Schiff noch immer vom Eis umschlossen war, traf man am 24. Februar 1874 die Entscheidung, es zu verlassen und die Expeditionsgruppe zu Fuß nach Süden zu retten. Am 20. Mai 1874 verließ die Mannschaft die noch immer unbeweglich festgefrorene „Admiral Tegetthoff“. Ein abenteuerlicher Rückzug führte die Mannschaft schließlich zurück nach Nordnorwegen. Feste Eisflächen und mobiles Treibeis behinderten das Vorwärtskommen der Boote. Erst nach drei Monaten erreichten die Polarfahrer am 15. August 1874 offenes Meer. Mit Hilfe eines russischen Fangschiffes erreichte die Mannschaft am 3. September 1874 das norwegische Vardø.

Die „Admiral Tegetthoff“ blieb im Polareis eingeschlossen. Dass beinahe die gesamte Mannschaft der „Tegetthoff” heil nach Hause zurückkehrte, verdankt sie der Umsicht der beiden Expeditionsleiter. Deren weiteres Leben war eher traurig und endete ohne öffentliche Anerkennung. Auch August Petermann, dessen Annahme eines offenen Polarmeeres die Expedition nicht bestätigen konnte, schlitterte in eine schwere Depression und beging 1878 Selbstmord. Die Bilder der im Eis eingeschlossenen, beschädigten „Admiral Tegetthoff“ wurden allgemein bekannt.

Für den Übergang vom 20. zum 21. Jahrhundert können weitere Forschungsarbeiten angeführt werden, die den Namen ,Tegetthoff“ wieder in Erinnerung riefen. Mitte der 1990er Jahre wurde eine Studie über Fernerkundungskartographie von Franz-Josef-Land durchgeführt (KosTKA 1997a), bei der österreichische und russische Fachleute zusammen arbeiteten. Die Arbeit mit unterschiedlichen Themenstellungen zum gesamten Archipel erfolgte mit russischen und westlichen Satellitenbildern und führte u. a. zu einer mehrfärbigen kartographischen Darstellung des gesamten Inselbereiches im Maßstab 1:600.000. Das Namengut ist in Russisch und Englisch angegeben. In dieser Studie ist auch eine Übersichtskarte von Franz-Josef-Land mit der Hall Insel und dem „Kap Tegetthoff“ enthalten (Abb. 3). Von den Mitautoren der Sammelpublikation, die im Ergänzungsheft 293 zu Petermanns Geographischen Mitteilungen beim Verlag Justus Perthes in Gotha herausgebracht wurde, widmete sich der Österreicher Josef Breu dem Namengut der Hall Insel. Auf Tegetthoff selbst und das nach ihm benannte Kap wird mehrmals Bezug genommen (BREU in KosTKA 1997a).

Im Jahr 2005 wurde unter der Vorbereitung und Durchführung von Christof Höbenreich eine Payer-Weyprecht-Gedächtnisexpedition erfolgreich durchgeführt (HöBENREICH 2007). Im Zentrum stand eine Schi- und Schlittenreise in Erinnerung an die drei überwiegend per Schlitten durchgeführten Erkundungsreisen von Julius Payer auf Franz-Josef-Land im Jahr 1874, um die historische Leistung der Pioniere zu würdigen. Die österreichisch-russische Expeditionsmannschaft bestand aus zwei österreichischen und zwei russischen Teilnehmern.

Selbstverständlich zählt auch das Kap Tegetthoff mit seinen beiden steil aufragenden Felsnadeln an der Südspitze der Hall Insel zu den arktischen Höhepunkten der heutigen globalen Interessen. Auch in russischen Kartenblättern der Gegenwart findet man den Namen Kap Tegetthoff [Mys tegetgofa].

\section{Globalität-Mobilität der Gegenwart am lokalen Beispiel des „Tegetthoff-Steines“}

Seit der zweiten Hälfte des 19. Jahrhunderts, der Zeit, in der Tegetthoff lebte und wirkte, haben sich die Umwelt und damit auch der Lebensraum stark gewandelt. Die industrielle Produktion und 


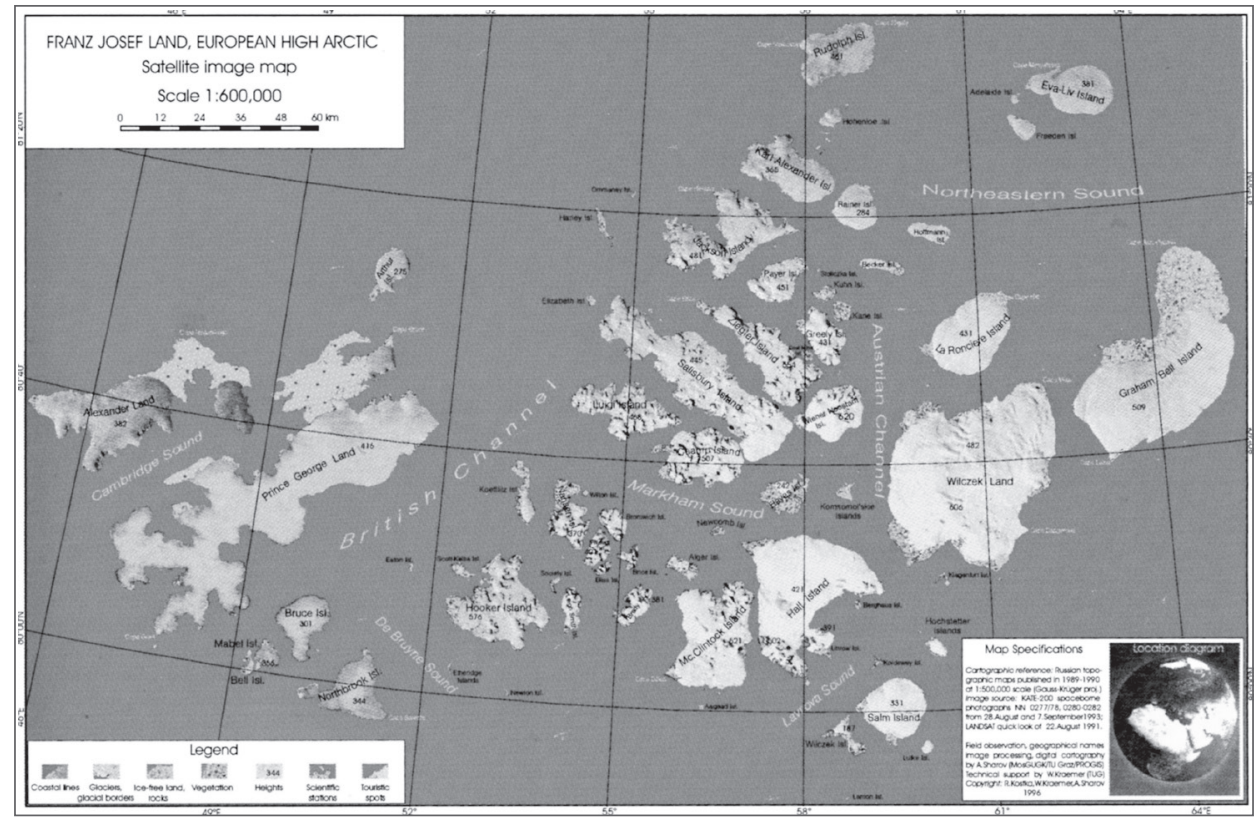

Quelle: KostKa 1997a, Kartenbeilage

Abb. 3: Übersichtskarte von Franz-Josef-Land (Schwarz-Weiß-Verkleinerung) mit der Hall Insel Kap Tegetthoff im Süden

der Massenkonsum prägen die Gesellschaft der Stadtmenschen der Gegenwart. Nicht zuletzt durch die gegenwärtigen sowie die in Weiterentwicklung befindlichen technischen Möglichkeiten entstehen neue Bedürfnisse und Interessen im globalen Umfeld.

Die Begriffe von Globalität und Mobilität sind in der heimatlichen Region von Wilhelm von Tegetthoff bis in die Gegenwart erhalten geblieben, ihre Interpretation richtet sich aber nach dem aktuellen Zeitgeist; das betrifft seinen Lieblingshafen Triest, seine Geburtsstadt Marburg an der Drau, die Weingüter bei Ehrenhausen an der Mur. Es sind Erinnerungen an seine Brieffreundin Freifrau von Lutterot. Schließlich nördlich davon die anerkannte Studentenstadt Graz bis nach St. Radegund am Fuß des Schöckl, wo 1870, also noch vor seinem Ableben, das Denkmal des ,Tegetthoff-Steines“ errichtet wurde. Das lokale Beispiel des Wandels soll durch die Gegenüberstellung von Tradition und globalisierter Form der Lebensgewohnheiten des modernen Stadtmenschen mit dem Tegetthoff-Stein im Bereich St. Radegund, Schöckl, kurz skizziert werden (GRÖLLER 2016).

Der „Tegetthoff-Stein“ (Abb. 4), der sich seit seiner Errichtung kaum verändert hat, befindet sich, wie eingangs erwähnt, an einer aussichtsreichen Stelle am Südabhang des Schöckl. Der Platz mit einem weiten Ausblick in die Südoststeiermark vom Kulm bis zum Novystein diente dem die Öffentlichkeit scheuenden Tegetthoff zur Ruhe und Erholung. Heute liegen die Interessen an diesem Bereich vom Schöckl bis St. Radegund, zumindest an Wochenenden, in der Touristik und im Sport, vom Wandern und Bergsteigen - Weitwanderwege und Wallfahrtswege - bis zur Benützung der Seilbahn. Auch unterschiedliche Varianten der Mobilität findet man in dem angegebenen Gebiet in Hanglage. Von den barrierefreien Wanderrouten reichen sie über unterschiedliche Radfahrmöglichkeiten - Radrennen und Mountainbiking - und über eine Sommerrodelbahn bis zu den motorbetrie- 
benen Varianten. Für Privat- und öffentlichen Verkehr reichen die Möglichkeiten vom Parkhaus bis zur asphaltierten Bergstraße. Besondere Beachtung finden dabei aber die Einschränkungen für diese Fortbewegungsart. Neben Einbahnregelung und Geschwindigkeitsbeschränkung reichen sie bis zu Fahrverboten. Für die angesprochenen Sportinteressierten reichen die Möglichkeiten vom Flugsport mit Startrampe und Landewiese für Hängegleiter und Drachenflieger bis zu den Klettervarianten in einem Klettergarten im Nadelwald oder Boulderfelsen. Die Dynamik der gegenwärtigen Interessen mit globalisierten Elementen trifft mit der Idee des Kurortes - Rastplatz, Meditationsweg, Heilwasseranwendung wie zur Zeit von Tegetthoff - zusammen.

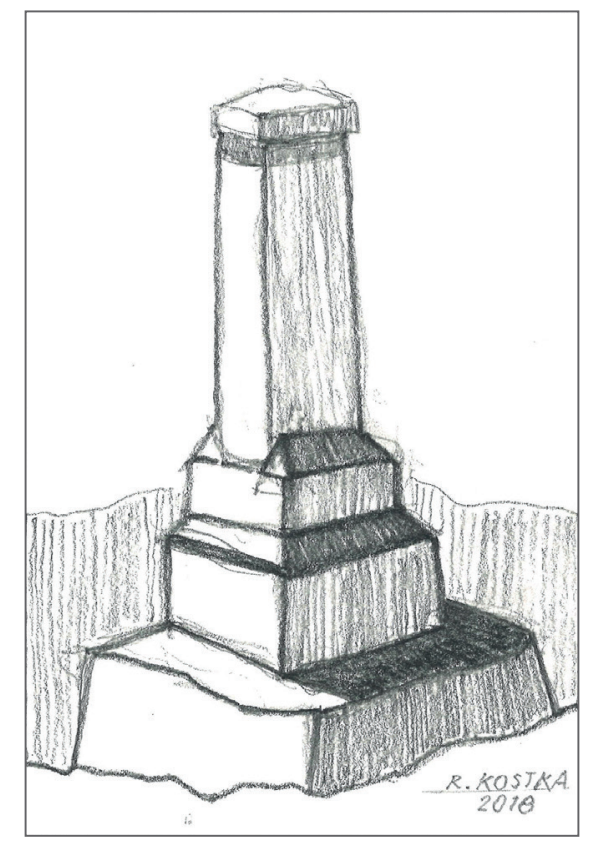

Quelle: KostKa 2018, Grafik (eigener Entwurf)

Abb. 4: Der Tegetthoff-Stein, Gedenkstein für Wilhelm von Tegetthoff, über dem Kurort St. Radegund am Abhang des Schöckl, errichtet 1870.

Die Umweltveränderungen bis zur Gegenwart, die unterschiedlichen Formen des Wandels sind als Tatsachen zu akzeptieren. Das Objekt oder die Position einer Gedenkstätte wie der „Tegetthoff-Stein“ sollten jedoch möglichst unverändert erhalten bleiben und lediglich in die gegenwärtige Situation eingefügt werden. Gedenkstätten sollten in ehrenden Gedanken an anerkannte Persönlichkeiten durch ein Objekt, wie erwähnt, oder zur Erinnerung an ein besonderes Ereignis durch die Position einer bleibend definierten Geländeform errichtet bzw. ausgewiesen werden, wie wir sie bei Wilhelm von Tegetthoff antreffen.

Durch Entwicklung und Fortschritt wird auch in Zukunft der Gegensatz zwischen traditioneller Gedenkstätte und den Auswirkungen der globalen Mobilität bestehen bleiben. In einer Welt mit fortschreitender Globalisierung und variantenreicher Mobilität wird man diese Situation zur Kenntnis zu nehmen haben. 


\section{Literaturverzeichnis}

BASCh-Ritter R. (1987): Österreich auf allen Meeren, Geschichte der k.(u.)k. Kriegsmarine von 1382 bis 1918. Graz - Wien - Köln, Verlag Styria.

GRÖLlER H. D. (2016): St. Radegund. Ein steirischer Kurort und seine Geschichte, Ortschronik, Band 1, 2. St. Radegund, Tourismusverband.

Heeresgeschichtliches Museum Wien, Militärhistorisches Institut (Hrsg.) (1996): Die Schrecken des Eises und der Finsternis. Wien, Heeresgeschichtliches Museum.

HöBenReICH Ch. (2007): Expedition Franz Josef Land, in der Spur der Entdecker nach Norden. München, Frederking \& Thaler Verlag.

JANATA A. (1989): Jemen - Im Land der Königin von Saba. Wien, Museum für Völkerkunde.

KostKa R. (1997a): The Franz Josef Land Archipelago - Remote Sensing and Cartography (= Petermanns Geographische Mitteilungen, Ergänzungsheft 293). Gotha, Justus Perthes Verlag. Mit den Kartenbeilagen „Franz Josef Land Archipelago“, „Russian Arctic“, im Maßstab 1:600.000, und Hall Island - Cape Tegetthoff, 1:50.000. Namengut mit Österreich-Bezug.

KostKa R. (1997b): Franz Josef Land. Studien zur Kartographie des nördlichsten Archipels Europas. In: Mitteilungen der Österreichischen Geographischen Gesellschaft, 139, S. 313-326.

KostKa R. (2016): Johann Nepomuk Graf Wilczek und die österreichische Arktisforschung. In: Mitteilungen der Österreichischen Geographischen Gesellschaft, 158, S. 320-333.

PAYer J., Weyprecht C. (1874): Oesterreichisch-ungarische Nordpol-Expedition. 1872 bis 1874. In: Mittheilungen der k.k. Geographischen Gesellschaft in Wien, 17, S. 389-417.

WALLisCh M. (2006): Expeditionen der k.k. Geographischen Gesellschaft in Wien. In: KretsChmER I., FASChING G. (Hrsg.): Österreich in der Welt - die Welt in Österreich. Chronik der Österreichischen Geographischen Gesellschaft, 150 Jahre (1856-2006). Wien, Österreichische Geographische Gesellschaft, S. 269-288.

WAWrik F., Fischer H., ZeILINGER E. (1986): Kartographische Kostbarkeiten. Wien, Österreichische Nationalbibliothek. 